Annals of Pure and Applied Mathematics

Vol. 16, No. 2, 2018, 471-477

ISSN: 2279-087X (P), 2279-0888(online)

Published on 29 March 2018

Annals of

www.researchmathsci.org

DOI: http://dx.doi.org/10.22457/apam.v16n2a25

Pure and Applied

Mathematics

\title{
Solutions of the Diophantine Equation $2^{x}+p^{y}=z^{2}$ When $p$ is Prime
}

Nechemia Burshtein

117 Arlozorov Street, Tel Aviv 6209814, Israel

Email: anb17@netvision.net.il

Received 16 March 2018; accepted 29 March 2018

Abstract. In this article, we consider the Diophantine equation $2^{x}+p^{y}=z^{2}$ when $p=4 N+3$ and $p=4 N+1$ are primes. The values $x, y, z$ are positive integers. For each prime, all the possibilities for solutions are investigated. All cases of no-solutions, as well as cases of infinitely many solutions are determined. Whenever the number of solutions for $p=4 N+3 / p=4 N+1$ is finite, we establish the respective connection between this number to all Mersenne Primes / Fermat Primes known as of 2018. Numerical solutions of various cases are also exhibited.

Keywords: Diophantine equations, Catalan's Conjecture, Mersenne, Fermat Primes

AMS Mathematics Subject Classification (2010): 11D61

\section{Introduction}

The field of Diophantine equations is ancient, vast, and no general method exists to decide whether a given Diophantine equation has any solutions, or how many solutions. In most cases, we are reduced to study individual equations, rather than classes of equations.

The literature contains a very large number of articles on non-linear such individual equations involving primes and powers of all kinds. Among them are for example $[1,5$, $9,10]$.

The famous general equation

$$
p^{x}+q^{y}=z^{2}
$$

has many forms, in particular when $p=2[2,3,9,11]$.

In this article we consider the equation

$$
2^{x}+p^{y}=z^{2}
$$

and in our discussion, we utilize Catalan's Conjecture, Mersenne Primes and Fermat Primes.

In 1844 E. C. Catalan conjectured: The only solution in integers $r>0, s>0$, $a>1, b>1$ of the equation

is $r=b=3$ and $s=a=2$.

$$
r^{a}-s^{b}=1
$$

The conjecture was proven by P. Mihăilescu [6] in 2002. 


\section{Nechemia Burshtein}

The interest in numbers $2^{n}-1$ being primes dates to antiquity. When $n$ is composite, $2^{n}-1$ is not a prime. In 1644, Mersenne published a list of 11 primes for which he claimed that $2^{n}-1$ is a prime. The first Mersenne Primes [4] are 3, 7, 31, 127. The condition that $n$ be a prime is a necessary but not a sufficient condition for the primality of $2^{n}-1\left(2^{11}-1=2047=23 \cdot 89\right)$. The search for primes $n$ for which $2^{n}-1$ is a prime continued over the years. On January 3, 2018 the largest $50^{\text {th }}$ known Mersenne Prime $2^{77,232,917}-1$ was discovered by GIMPS [4]. It has $23,249,425$ digits and is the largest prime known to mankind [4].

A Fermat Prime is a prime of the form $2^{n}+1$ where $n$ is a power of 2. As of 2018 [12], only five Fermat Primes are known. For $n=1,2,4,8,16$, these primes are $\{3,5,17,257,65537\}$.

In Section 2 we find all the solutions of $2^{x}+p^{y}=z^{2}$ when $p=4 N+3$ is prime. In Section 3 we find solutions of the above equation when $p=4 N+1$ is prime.

2. All the solutions of $2^{x}+p^{y}=z^{2}$ when $p=4 N+3$ is prime

In this section, we discuss all the cases of equation (1) when $p=4 N+3$ is prime. In each case, we determine all the solutions. This is done in Theorems 2.1 and Theorem 2.2 which are self-contained. We also demonstrate some numerical solutions.

Theorem 2.1. Suppose that $p=4 N+3(N \geq 0)$ is prime. If $y=2 n+1$ is odd in $2^{x}$ $+p^{y}=z^{2}$, then:

(a) For $x=1$ and $y=1 \quad(n=0)$, equation (1) has infinitely many solutions.

(b) For $x=1$ and $y>1$, equation (1) has no solutions.

(c) For $x>1$ and $y \geq 1$, equation (1) has no solutions.

Proof: In $2^{x}+p^{y}=z^{2}$ the integer $z^{2}$ is odd, hence $z$ is odd. Denote $z=2 U+1$. Then $z^{2}=(2 U+1)^{2}=4 U(U+1)+1$.

(a) Suppose that $x=1$ and $y=1$. We have from equation (1)

$$
2+p=z^{2}
$$

From equation (2) we then obtain

$$
2+(4 N+3)=4 U(U+1)+1=z^{2} .
$$

Thus, $p=4 N+3=4 U(U+1)-1$. Evidently, equation (2) is now

$$
2+(4 U(U+1)-1)=(2 U+1)^{2}
$$

an identity valid for infinitely many values $U \geq 1$. Hence, equation (2) has infinitely many solutions as asserted.

For the first five values $U=1,2,3,4,6$, and the convenience of the readers, we exhibit the five solutions of equation (2) as follows:

$\begin{array}{ll}\text { Solution 1. } 1 . & 2^{1}+7^{1}=3^{2} \\ \text { Solution 2. } & 2^{1}+23^{1}=5^{2} \\ \text { Solution 3. } & 2^{1}+47^{1}=7^{2} \\ \text { Solution 4. } & 2^{1}+79^{1}=9^{2} \\ \text { Solution 5. } & 2^{1}+167^{1}=13^{2} .\end{array}$

We note that Solution 1 has already been obtained in [3]. 
Solutions of the Diophantine Equation $2^{x}+p^{y}=z^{2}$ when $p$ is Prime

(b) Suppose that $x=1$ and $y>1$ is odd. The equation $2^{x}+p^{y}=z^{2}$ is

$$
2+p^{y}=z^{2} \text {. }
$$

From (3) it follows that $p^{y}+1=z^{2}-1=4 U(U+1)$ or

$$
p^{2 n+1}+1=4 U(U+1), \quad n \geq 1 \text {. }
$$

Equality (4) yields

$$
p^{2 n+1}+1^{2 n+1}=(p+1)\left(p^{2 n}-p^{2 n-1} \cdot 1^{1}+\cdots+1^{2 n}\right)=4 U(U+1) .
$$

In (5), the factor $\left(p^{2 n}-p^{2 n-1} \cdot 1^{1}+\cdots+1^{2 n}\right)$ is odd for all values $p$. Therefore $4 \mid(p+1)$, and hence from $(5)$

$$
(N+1)\left((4 N+3)^{2 n}-(4 N+3)^{2 n-1} \cdot 1^{1}+\cdots+1^{2 n}\right)=U(U+1) .
$$

The even term $U(U+1)$ in (6) is the product of two consecutive integers, and hence $N$ is odd. It is seen that equality (6) does not hold.

Therefore, equation (3) has no solutions when $y>1$ is odd.

(c) Suppose that $x>1$ and $y \geq 1$ is odd. Then $2^{x}+p^{y}=z^{2}$ is

$$
2^{x}+p^{2 n+1}=z^{2}, \quad n \geq 0 .
$$

For all integers $x>1,2^{x}=4 \cdot 2^{x-2}$. The integer $z^{2}$ has the form $4 T+1$. It is easily verified for every value $n \geq 0$, that $p^{2 n+1}$ is of the form $4 M+3$.

In (7), the left-hand side has the form

$$
2^{x}+p^{2 n+1}=4 \cdot 2^{x-2}+(4 M+3)=4\left(2^{x-2}+M\right)+3,
$$

whereas the right-hand side of (7) is of the form

$$
z^{2}=4 T+1
$$

The two sides of equation (7) contradict each other. Therefore, for each prime $p$, there do not exist integers $x, y$ and $z$ which satisfy equation (1).

This concludes the proof of Theorem 2.1.

Theorem 2.2. Suppose that $p=4 N+3(N \geq 0)$ is prime. If in $2^{x}+p^{y}=z^{2} y=2 n$ is even, then:

(a) For $n=1$, equation (1) has 50 solutions.

(b) For $n>1$, equation (1) has no solutions.

Proof: Equation (1) is now

From (8) we obtain

$$
2^{x}+p^{2 n}=z^{2}, \quad n \geq 1 .
$$

Denote

$$
2^{x}=z^{2}-p^{2 n}=z^{2}-\left(p^{n}\right)^{2}=\left(z-p^{n}\right)\left(z+p^{n}\right) .
$$

$$
z-p^{n}=2^{\alpha}, \quad z+p^{n}=2^{\beta}, \quad \alpha<\beta, \quad \alpha+\beta=x .
$$

Hence from (9)

$$
2 p^{n}=2^{\beta}-2^{\alpha}=2^{\alpha}\left(2^{\beta-\alpha}-1\right) .
$$

It clearly follows from (10) that $\alpha=1$, and therefore

$$
2^{\beta-1}-1=p^{n} \text {. }
$$

(a) Suppose that $n=1$. Then (11) yields $2^{\beta-1}-1=p$ which may be a Mersenne Prime. Every Mersenne Prime is of the form $4 N+3$ as required by our supposition, and therefore is a solution of equation (1). It is known [4], that there are 50 Mersenne Primes, of which by January 2018 the largest is equal to $p=2^{77,232,917}-1$ being the 


\section{Nechemia Burshtein}

$50^{\text {th }}$ prime. Hence, equation (1) has 50 solutions up to $2^{77,232,917}-1$ inclusive, as asserted. Each such solution is of the form $2^{1+\beta}+\left(2^{\beta-1}-1\right)^{2}=z^{2}$.

For the convenience of the readers, we demonstrate the first three solutions of equation (8) when $n=1$.

Solution 6. $(\beta-1=2, \quad p=3)$

Solution 7. $\quad(\beta-1=3, \quad p=7)$

Solution 8. $\quad(\beta-1=5, \quad p=31)$

$$
\begin{aligned}
& 2^{4}+3^{2}=5^{2} \\
& 2^{5}+7^{2}=9^{2} \\
& 2^{7}+31^{2}=33^{2}
\end{aligned}
$$

We remark that Solution 7 has already been obtained in [2].

(b) Suppose that $n>1$. It clearly follows from (11) that $\beta-1>1$. Therefore, by Catalan's Conjecture the equation $2^{\beta-1}-p^{n}=1$ has no solutions. Thus, for all values $n>1$ equation (1) has no solutions as asserted.

This concludes the proof of Theorem 2.2.

Concluding remark. It is now known that there exist 50 Mersenne Primes. In Theorem 2.2 part (a), when $y=2$ and $p$ is a Mersenne Prime, it has been established that $2^{x}+p^{2}=z^{2}$ has exactly 50 known solutions. Almost all of these solutions are achieved by a computer. Additional solutions of the above equation solely depend on finding more Mersenne Primes.

\section{Solutions of $2^{x}+p^{y}=z^{2}$ when $p=4 N+1$ is prime}

In this section, $p=4 N+1$ is prime, and all cases of equation (1) are considered. This is done in the following Theorems $3.1-3.3$ each of which is self-contained. Numerical solutions are also exhibited.

Theorem 3.1. Suppose that $p=4 N+1$ is prime. If $y=2 n$ is even in $2^{x}+p^{y}=z^{2}$, then for all values $x$, the equation

$$
2^{x}+p^{2 n}=z^{2}, \quad n \geq 1
$$

has no solutions.

Proof: In $2^{x}+p^{y}=z^{2}$ the integer $z^{2}$ is odd, hence $z$ is odd. Denote $z=2 U+1$. Then $z^{2}=(2 U+1)^{2}=4 U(U+1)+1$.

From (12) we have

Denote

$$
2^{x}=z^{2}-p^{2 n}=z^{2}-\left(p^{n}\right)^{2}=\left(z-p^{n}\right)\left(z+p^{n}\right)
$$

From (13) it follows that

$$
z-p^{n}=2^{\alpha}, \quad z+p^{n}=2^{\beta}, \quad \alpha<\beta, \quad \alpha+\beta=x .
$$

$$
2 \cdot p^{n}=2^{\beta}-2^{\alpha}=2^{\alpha}\left(2^{\beta-\alpha}-1\right) .
$$

Equality (14) implies that $\alpha=1$. Thus (14) yields

$$
p^{n}=2^{\beta-1}-1 \text {. }
$$

By (13) $\beta>\alpha=1$, and from (15) $\beta=2$ is impossible. Thus $\beta>2$. Since 
Solutions of the Diophantine Equation $2^{x}+p^{y}=z^{2}$ when $p$ is Prime

$p=4 N+1$, therefore for each and every $n \geq 1, p^{n}$ is of the form $4 M+1$. For all values $\beta>2$, the right-hand side of (15) is of the form $4 V+3$. The two sides of (15) contradict each other, and therefore (15) is impossible.

Hence, equation (12) has no solutions as asserted.

Theorem 3.2. Suppose that $p=4 N+1$ is prime. If in $2^{x}+p^{y}=z^{2} x=2 t$ is even and $y=2 n+1$ is odd, then the equation

has:

$$
2^{2 t}+p^{2 n+1}=z^{2}, \quad t \geq 1, \quad n \geq 0
$$

(a) No solutions for all values $n \geq 1$.

(b) 4 solutions when $n=0$.

Proof: (a) Suppose that $n \geq 1$. From (16) we obtain

$$
p^{2 n+1}=z^{2}-2^{2 t}=z^{2}-\left(2^{t}\right)^{2}=\left(z-2^{t}\right)\left(z+2^{t}\right) \text {. }
$$

Denote

$$
z-2^{t}=p^{\alpha}, \quad z+2^{t}=p^{\beta}, \quad \alpha<\beta, \quad \alpha+\beta=2 n+1 .
$$

From (17) it follows that

$$
2 \cdot 2^{t}=p^{\beta}-p^{\alpha}=p^{\alpha}\left(p^{\beta-\alpha}-1\right) .
$$

Equality (18) implies that $\alpha=0$, and hence (18) yields

$$
2^{t+1}=p^{\beta}-1 \text {. }
$$

From (19) and Catalan's Conjecture, $p^{\beta}-2^{t+1}=1$ has the only solution $p=3(\beta=2, t=2)$. But $p=3$ is not of the form $4 N+1$. Therefore, for all values $t$, $n \geq 1$ and $p=4 N+1$, the equation $2^{2 t}+p^{2 n+1}=z^{2}$ has no solutions.

This completes the proof of part (a).

(b) Suppose that $n=0$. When $\alpha=0$ in (17) then $\beta=1$. Therefore (19) yields

$$
2^{t+1}+1=p \text {. }
$$

In (20), the prime $p$ is known as Fermat Prime, where $(t+1)$ must be a power of 2 . To the present day [12], only five Fermat Primes are known. These are

$$
\{3,5,17,257,65537\} \text {. }
$$

The prime $p=3$ cannot be used in (16), since it is not of the form $p=4 N+1$. Note that for all $t \geq 1$, the prime $p$ in (20) is of the form $4 N+1$. Thus, any Fermat Prime $>3$ is a solution of (16). Therefore, when $t \geq 1, n=0$ and $p=4 N+1$, the equation $2^{2 t}+p^{2 n+1}=z^{2}$ has only 4 solutions.

The 4 solutions are presented as follows.

$\begin{array}{llrlr}\text { Solution 9. } & 2^{2}+ & 5^{1} & & 3^{2} \\ \text { Solution 10. } & 2^{6}+ & 17^{1} & = & 9^{2} \\ \text { Solution 11. } & 2^{14}+257^{1} & = & 129^{2} \\ \text { Solution 12. } & 2^{30}+65537^{1} & = & 32769^{2} .\end{array}$

This concludes the proof of Theorem 3.2.

Theorem 3.3. Suppose that $p=4 N+1$ is prime. If in $2^{x}+p^{y}=z^{2} \quad x=2 t+1$ is odd and $y=2 n+1$ is odd, then the equation 
has:

$$
\begin{aligned}
& \text { Nechemia Burshtein } \\
& 2^{2 t+1}+p^{2 n+1}=z^{2}, \quad t \geq 0, \quad n \geq 0
\end{aligned}
$$

(a) No solutions when $t=0$ and $n=0$.

(b) Infinitely many solutions when $t \geq 1$ and $n=0$.

(c) No solutions when $t=0$ and $n \geq 1$.

(d) At least one solution when $t \geq 1$ and $n \geq 1$.

Proof: (a) Suppose that $t=0$ and $n=0$. From (21) we obtain

$$
2^{1}+p^{1}=2+(4 N+1)=4 N+3=z^{2}
$$

which is impossible since $z^{2}$ is odd and has the form $z^{2}=4 T+1$. In case (a), equation (21) has no solutions as asserted.

(b) Suppose that $t \geq 1$ and $n=0$. It is then easily seen that infinitely many solutions of (21) exist. Some of these are demonstrated here.

$\begin{array}{lll}\text { Solution 13. } & 2^{3}+17^{1}=5^{2} . \\ \text { Solution 14. } & 2^{5}+89^{1}=11^{2} . \\ \text { Solution 15. } & 2^{7}+41^{1}=13^{2} . \\ \text { Solution 16. } & 2^{9}+113^{1}=25^{2} . \\ \text { Solution 17. } & 2^{11}+353^{1}=49^{2} .\end{array}$

It is noted that more than one solution may exist for a value $t$. For instance: $2^{3}+41^{1}=7^{2}, 2^{7}+97^{1}=15^{2}$, and so on. Case (b) is complete.

(c) Suppose that $t=0$ and $n \geq 1$. We have from (21)

$$
2^{1}+p^{2 n+1}=z^{2} \text {. }
$$

The form of $z^{2}$ is equal to $4 T+1$, and for all $n \geq 1, p^{2 n+1}$ has the form $4 M+1$. Then, the left-hand side of (22) is of the form $4 M+3$, whereas the right-hand side has the form $4 T+1$. This contradicts the existence of (22). Hence, the equation $2+p^{2 n+1}=z^{2}$ has no solutions as asserted.

(d) Suppose that $t \geq 1$ and $n \geq 1$. We obtain

$$
2^{2 t+1}+p^{2 n+1}=z^{2}, \quad t \geq 1, \quad n \geq 1
$$

Then $2^{2 t+1}=4 \cdot 2^{2 t-1}, p^{2 n+1}=(4 N+1)^{2 n+1}$, and $z^{2}$ equals to $4 U(U+1)+1$. By the Binomial Theorem, the expansion of $(4 N+1)^{2 n+1}$ has $(2 n+2)$ terms. The first $(2 n+$ 1) terms are each a multiple of $(4 N)$, the $(2 n+2)^{\text {th }}$ term is equal to 1 . Denote the sum of the $(2 n+1)$ terms by $(4 N) M$ where $M$ is odd. Then, from (23) we have

which after simplifications yields

$$
4 \cdot 2^{2 t-1}+(4 N) M+1=4 U(U+1)+1
$$

$$
2^{2 t-1}+N M=U(U+1)
$$

The value $U(U+1)$ is a product of two consecutive integers and is even. Therefore, $N$ is even and denote $N=2 R, U(U+1)=2 W$. From (24) we then obtain

$$
2^{2 t-2}+R M=W \text {. }
$$

In (25), if $t>1$ then $R$ and $W$ are of the same parity, whereas when $t=1, R$ and $W$ are of a different parity. The process of finding solutions from here on presents great difficulties, and we shall not pursue this matter any further.

However, the following values $t=3, p=17, n=1, z=71$ when substituted in (23) yield the solution: 
Solutions of the Diophantine Equation $2^{x}+p^{y}=z^{2}$ when $p$ is Prime

Solution 18.

$$
2^{7}+17^{3}=71^{2} \text {. }
$$

Equivalently, we then have in (25)

$$
2^{2 t-2}=2^{4}, \quad R=2, \quad M=307, \quad W=630 .
$$

The proof of Theorem 3.3 is now complete.

Concluding remark. It is noted that $p=17$ is the third known Fermat Prime. The first Fermat Prime 3 is not of the form $4 N+1$. For the second Fermat Prime 5, all powers of 5 have a last digit equal to 5 . When added an odd power of 2 whose last digit is either 2 or 8 , it follows that in $2^{2 t+1}+5^{2 n+1}=z^{2}, \quad z^{2}$ has a last digit which is respectively either 7 or 3 . Since a square never has a last digit which is either 7 or 3 , it follows that the above equation has no solutions.

A solution of $2^{2 t+1}+p^{2 n+1}=z^{2}$ if such exists with either Fermat Prime 257 or 65537 requires the aid of a computer. Moreover, from [12], finding more Fermat Primes has a very low expectation.

Conjecture. Except for $p=5,17$, for all other primes $p$ either Fermat Primes or not, we conjecture that no solutions exist for $2^{2 t+1}+p^{2 n+1}=z^{2}$ when $t \geq 1, n \geq 1$.

If indeed the answer is affirmative, then the above equation has exactly one solution when $p=17$, namely Solution 18. Moreover, it then follows in Section 3 that all the solutions of $2^{x}+p^{y}=z^{2}$ when $p=4 N+1$ have been established.

\section{REFERENCES}

1. N. Burshtein, On the diophantine equation $p^{x}+q^{y}=z^{2}$, Annals of Pure and Applied Mathematics, 13 (2) (2017) 229 - 233.

2. N.Burshtein, On the diophantine equation $2^{2 x+1}+7^{y}=z^{2}$, Annals of Pure and Applied Mathematics, 16 (1) (2018) 177 - 179.

3. N.Burshtein, All the solutions to an open problem of S. Chotchaisthit on the diophantine equation $2^{\mathrm{x}}+p^{y}=z^{2}$ when $p$ are particular primes and $y=1$, Annals of Pure and Applied Mathematics, 16 (1) (2018) 31 - 35.

4. GIMPS Great Internet Mersenne Prime Search, GIMPS Finding World Record Primes.

5. Md.A.A.Khan, A.Rashid and Md. S.Uddin, Non-negative integer solutions of two diophantine equations $2^{x}+9^{y}=z^{2}$ and $5^{x}+9^{y}=z^{2}$, Journal of Applied Mathematics and Physics, 4 (2016) $762-765$.

6. P.Mihăilescu, Primary cyclotomic units and a proof of Catalan's conjecture, J. Reine Angew. Math. 572 (2004) $167-195$.

7. B.Poonen, Some diophantine equations of the form $x^{n}+y^{n}=z^{m}$, Acta Arith., 86 (1998) 193-205.

8. B.Sroysang, On the diophantine equation $5^{x}+7^{y}=z^{2}$, Int. J. Pure Appl. Math., 89 (2013) 115-118.

9. B.Sroysang, More on the diophantine equation $8^{x}+19^{y}=z^{2}$, Int. J. Pure Appl. Math., 81 (4) (2012) $601-604$.

10. A.Suvarnamani, On the diophantine equation $p^{x}+(p+1)^{y}=z^{2}$, Int. J. Pure Appl. Math., 94 (5) (2014) $689-692$.

11. A.Suvarnamani, A.Singta and S.Chotchaisthit, On two diophantine equations $4^{x}+$ $7^{y}=z^{2}$ and $4^{x}+11^{y}=z^{2}$, Sci. Techno. RMUTT J., 1 (1) (2011) 25-28.

12. Wolfram MathWorld, Fermat Prime. 